Original Research Article

\title{
Evaluation of antioxidant potential of melatonin in periodontitis: a prospective clinic-biochemical study
}

\author{
Anagha Marawar ${ }^{1}$, Pramod Marawar ${ }^{2}$, D. H. Nandal ${ }^{1}$, Rahul Kunkulol ${ }^{1}$, Sandeep Narwane ${ }^{1 *}$
}

${ }^{1}$ Department of Pharmacology, ${ }^{2}$ Department of Periodontics, Rural Dental College, Loni, Ahmednagar, Maharashtra, India

Received: 02 April 2019

Revised: 03 April 2019

Accepted: 03 May 2019

*Correspondence to:

Dr. Sandeep Narwane,

Email: drsandeepnarwane1984

@gmail.com

Copyright: (C) the author(s), publisher and licensee Medip Academy. This is an openaccess article distributed under the terms of the Creative Commons Attribution NonCommercial License, which permits unrestricted noncommercial use, distribution, and reproduction in any medium, provided the original work is properly cited.

\begin{abstract}
Background: The present study was done with an objective to evaluate the effect of melatonin as an antioxidant in patients suffering from periodontitis.

Methods: This study was conducted in the Department of Periodontics, Rural Dental College, Loni. Patients of chronic periodontitis, of age between 18 to 65 years of either gender ready to give informed consent to participate in the study were included. Postoperative patients, patients having night duties, drivers and those using heavy machinery, pregnant women, lactating mothers, patients with any clinically significant systemic disease and patients on any other drugs were excluded from the study. Patients were divided into three Groups. Group A included patients who underwent SRP (Scaling and Root Planning) alone, Group B included patients who underwent SRP \& supplemented with Vitamin E 200 IU daily at night for 4 weeks. Group C included patients who underwent SRP \& supplemented with tablet melatonin $3 \mathrm{mg}$ daily at night for 4 weeks. Estimation of Malondialdehyde (MDA) for serum lipid peroxidation8, Superoxide dismutase (SOD) and Glutathione peroxidase (GPx) was done on day 0, day 30, day 60 and day 90.

Results: A total 240 patients were enrolled in the study. It was demonstrated that there was considerable oxidative stress in periodontitis patients, as established by high serum MDA levels, which was reduced significantly by melatonin reflecting its antioxidant potential. Pretreatment levels of SOD and GPx also were low, which were improved with the treatment of melatonin far better than with vitamin E.

Conclusions: Melatonin acts as an antioxidant in the patients of periodontitis which has positive effect on biochemical parameters of periodontitis, conferring a new facet to the management of periodontitis and an attempt to impede the disease progression.
\end{abstract}

Keywords: Antioxidant, Melatonin, Periodontitis, Vitamin E

\section{INTRODUCTION}

Periodontitis is regarded as an inflammatory lesion, mediated by complex host parasite interactions, that leads to the loss of connective tissue attachment to root surface cementum and adjacent alveolar bone. ${ }^{1}$

Tissue infiltration by polymorphonuclear leukocytes and monocytes and subsequent phagocytosis, features a burst of cyanide insensitive (i.e., non-mitochondrial) $\mathrm{O}_{2}$ consumption, which may be 10 or 20 times that of resting consumption. Oxygen uptake in neutrophils and macrophages is due to the action of a plasma-membranebound flavoprotein cytochrome $b_{245}$ NADPH oxidase system that increases NADPH production via the hexose monophosphate shunt and generates superoxide anion radicals, hydrogen peroxide, hydroxyl radicals, and hypochlorous acid, all capable of damaging either cell membranes or associated biomolecules. ${ }^{2}$ A homeostatic imbalance between ROS and antioxidant defense systems can trigger an oxidative stress response, which is believed to be related to periodontal destruction. ${ }^{3}$ Until now, 
primary clinical weapons against periodontal disease have been scaling and root planning (SRP), antibiotics and surgery. Antioxidants, if given, can act systemically to support the body's natural immune system.

Melatonin is a ubiquitous natural neurotransmitter like compound produced primarily by pineal gland. ${ }^{4}$ Melatonin is identified as a powerful direct free radical scavenger and indirect antioxidant. ${ }^{5}$ Melatonin reduces oxidative stress by several means. It is an active scavenger of both the highly toxic hydroxyl radical $(\mathrm{OH})$, produced by 3 electron reduction of oxygen and peroxy radical which is generated during unsaturated lipid peroxidation. Melatonin also stimulates some important antioxidant enzymes, i.e., superoxide dismutase (SOD), glutathione peroxidase (GPx) and glutathione reductase. ${ }^{6}$ Melatonin additionally may stimulate the proliferation and synthesis of type I collagen and bone formation. ${ }^{7}$ Furthermore, many studies have proved that salivary, melatonin level varies according to the degree of periodontal disease indicating that salivary melatonin may act to protect the body from external body insults. Therefore, melatonin supplementation, i.e., synthetic version of hormone melatonin may be potentially valuable in the treatment of periodontal diseases. ${ }^{6}$ Considering the above mentioned functions of melatonin, this study was designed to evaluate the ability of melatonin supplementation to raise the antioxidant capacity levels, to reduce lipid peroxidation and thereby to reduce the periodontal inflammation. This study is also aimed to compare the efficacy of melatonin and vitamin $\mathrm{E}$ supplementation as antioxidants in periodontal diseases. Present study was designed with an objective to determine the effect of melatonin and vitamin $\mathrm{E}$ on lipid peroxidation and antioxidant enzymes in periodontal disease.

\section{METHODS}

The present study was a prospective longitudinal type of study. The study commenced after the approval of Institutional ethical committee of Pravara Institute of Medical Sciences, Ahmednagar, Maharashtra, India. Patients satisfying the inclusion and exclusion criteria were included in the study after taking their written informed consent. Study was conducted at collaboration with the Department of Periodontics and Oral
Implantology, Rural Dental College and Department of Biochemistry, Rural Medical College and Hospital, Loni. The study was carried out during the period of January 2008 to December 2008. The subjects enrolled for this study were selected from the Out Patient Department of Periodontics, Rural Dental College, Loni, Maharashtra, India.

\section{Inclusion criteria}

Patients of chronic periodontitis, of age between 18 to 65 years of either gender ready to give informed consent to participate in the study were included.

\section{Exclusion criteria}

Postoperative patients, patients having night duties, drivers and those using heavy machinery, pregnant women, lactating mothers, patients with any clinically significant systemic disease and patients on any other drugs were excluded from the study. Grouping: Depending on the treatment received, the patients were divided into three groups as Group A included patients who underwent SRP (Scaling and Root Planning) alone, Group B who underwent SRP and supplemented with vitamin E $200 \mathrm{IU}$ daily at night for 4 weeks. Group C included Patients who underwent SRP and supplemented with tablet melatonin 3 $\mathrm{mg}$ daily at night for 4 weeks.

\section{Methodology}

The study participants visited on day 0 , day 30, day 60 and day 90 . During the baseline visit scaling and root planning was performed in all patients. During these visits the estimation of Malondialdehyde (MDA) for serum lipid peroxidation, Superoxide dismutase (SOD) and Glutathione peroxidase (GPx) was done. ${ }^{8-10}$ Statistical analysis was done by applying ' $Z$ ' test of difference between two sample means for comparison of MDA, SOD and GPx among the three groups.

\section{RESULTS}

In the present study, a total 240 patients were enrolled and evaluated in 2008.

Table 1: Age and sex wise distribution of the subjects under study.

\begin{tabular}{|lllllll|}
\hline Age in yrs & Group A & \multicolumn{3}{c|}{ Group B } & \multicolumn{3}{l|}{ Group C } \\
\hline & M & F & M & F & M & F \\
\hline$<40$ & $24(30 \%)$ & $6(7.5 \%)$ & $14(17.5 \%)$ & $23(28.7 \%)$ & $17(21.2 \%)$ & $11(13.7 \%)$ \\
\hline $40-50$ & $23(28.7 \%)$ & $15(18.7 \%)$ & $17(21.2 \%)$ & $12(15 \%)$ & $16(20 \%)$ & $17(21.2 \%)$ \\
\hline $50-60$ & $10(12.5 \%)$ & $2(2.5 \%)$ & $16(20 \%)$ & $12(15 \%)$ & $12(15 \%)$ & $7(8.75 \%)$ \\
\hline Total & $57(71.2 \%)$ & $23(28.7 \%)$ & $47(58.7 \%)$ & $33(41.2 \%)$ & $45(56.2 \%)$ & $35(43.7 \%)$ \\
\hline
\end{tabular}

Table 1 shows the age and sex wise distribution of the subjects in all 3 groups under study. All the three groups consisted of 80 subjects each. Group A consisted of $71.25 \%$ male and $28.75 \%$ female patients. Male patients in 
Group B were $58.75 \%$ and female $41.25 \%$. There were $56.25 \%$ male and $43.75 \%$ female patients in Group C.

From the Table 2 and Figure 1, it can be seen that, by applying ' $Z$ ' test of difference between two sample means, there is a highly significant decrease in mean values of MDA in group B when compared with group A at $3^{\text {rd }}$ visit $(\mathrm{p}<0.01)$, while there was significant rise during all visits except significant fall during visit 3 in MDA values when Group C and Group A were compared. Also, there was statistically significant rise in MDA during visits 1 and 2 respectively, when Group C was compared with Group B.

Table 2: Comparison of mean values of lipid peroxidation and antioxidants at four visits in all groups.

\begin{tabular}{|c|c|c|c|}
\hline & Group A (Mean \pm SD) & Group B $($ Mean \pm SD $)$ & Group C (Mean \pm SD) \\
\hline \multicolumn{4}{|c|}{ Malondialdehyde (MDA)- nmol/ml } \\
\hline Base line visit & $4.19 \pm 0.52$ & $4.71 \pm 1.14 *$ & $5.49 \pm 1.00 *, \#$ \\
\hline Visit $1^{\text {st }}$ & $3.96 \pm 0.58$ & $3.89 \pm 0.88$ & $4.57 \pm 1.18 *, \#$ \\
\hline Visit $2^{\text {nd }}$ & $4.18 \pm 0.58$ & $3.99 \pm 1.30$ & $4.50 \pm 1.29 *, \#$ \\
\hline Visit $3^{\text {rd }}$ & $4.21 \pm 0.60$ & $3.91 \pm 1.17 *$ & $3.61 \pm 1.27 *$ \\
\hline \multicolumn{4}{|c|}{ Superoxide dismutase (SOD)- Units/mg Hb } \\
\hline Base line visit & $2590.33 \pm 263.96$ & $2137.58 \pm 537.28 *$ & $2199.50 \pm 460.03 *$ \\
\hline Visit $1^{\text {st }}$ & $2571.62 \pm 208.27$ & $3435.42 \pm 521.72 *$ & $2862.51 \pm 873.11^{*, \#}$ \\
\hline Visit $2^{\text {nd }}$ & $2585.43 \pm 210.68$ & $2420.82 \pm 407.05^{*}$ & $2859.37 \pm 896.78^{*, \#}$ \\
\hline Visit $3^{\text {rd }}$ & $2543.94 \pm 198.48$ & $3468.10 \pm 425.84 *$ & $3428.52 \pm 904.43 *$ \\
\hline \multicolumn{4}{|c|}{ Glutathione peroxidase (GPx)- mgHb/min } \\
\hline Base line visit & $0.74 \pm 1.50$ & $0.70 \pm 0.12$ & $0.70 \pm 0.12$ \\
\hline Visit $1^{\text {st }}$ & $0.78 \pm 0.15$ & $1.72 \pm 0.47 *$ & $2.13 \pm 0.54 *, \#$ \\
\hline Visit $2^{\text {nd }}$ & $0.79 \pm 0.13$ & $1.90 \pm 0.53 *$ & $2.08 \pm 0.52 * \#$ \\
\hline Visit $3^{\text {rd }}$ & $0.77 \pm 0.12$ & $2.01 \pm 0.55^{*}$ & $2.02 \pm 0.53 *$ \\
\hline
\end{tabular}

*P $<0.01$ vs Group A (' $Z$ ' test of difference between two sample means), \# $\mathrm{P}<0.01$ vs Group B (' $Z$ ' test of difference between two sample means)

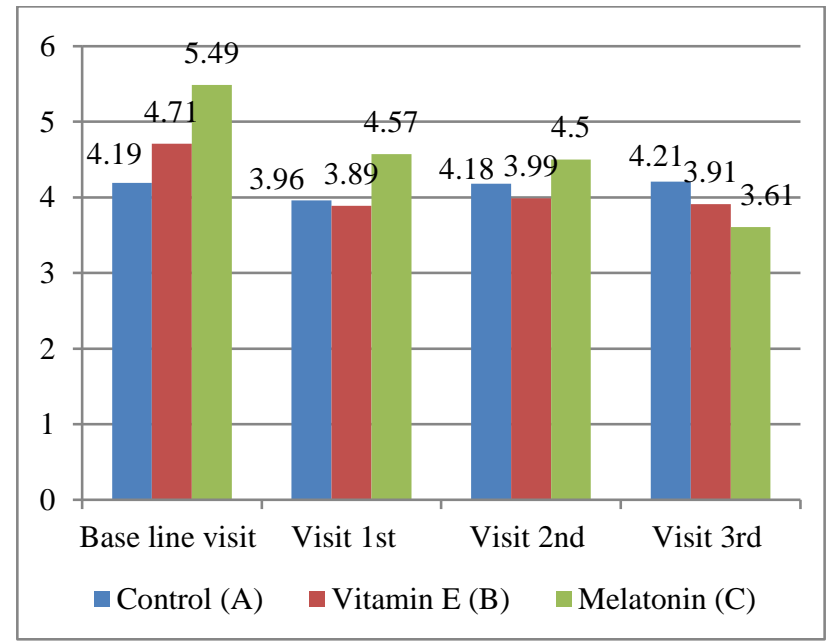

Figure 1: Comparison of mean values of malondialdehyde at four visits amongst all the groups.

On comparing the SOD values, there was a statistically significant increase during visit 1 and 3 and statistically significant decrease during visit 2 respectively, on comparing group B with group A. On comparing Group C with Group A, there was significant elevation of the SOD values during all visits. Also, the SOD values were significantly lower and higher during the visits 1 and respectively when group C was compared with Group B. (Table 2 and Figure 2).

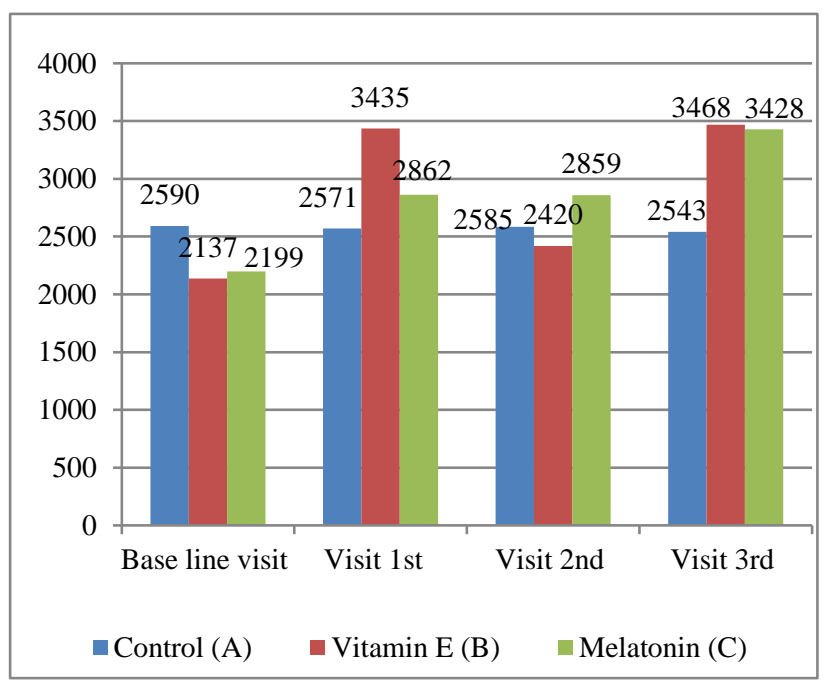

Figure 2: Comparison of mean values of superoxide dismutase at four visits amongst all the groups.

Table 2 and Figure 3 shows that GPx values were significantly lower during Visit 1 , while the values were significantly higher during visits 2 and 3 on comparing Group B with Group A. GPx values of all three visits were 
significantly higher when Group C was compared with Group A. Also, the GPx values were higher during visits 2 and_3 when Group C was compared with Group B.

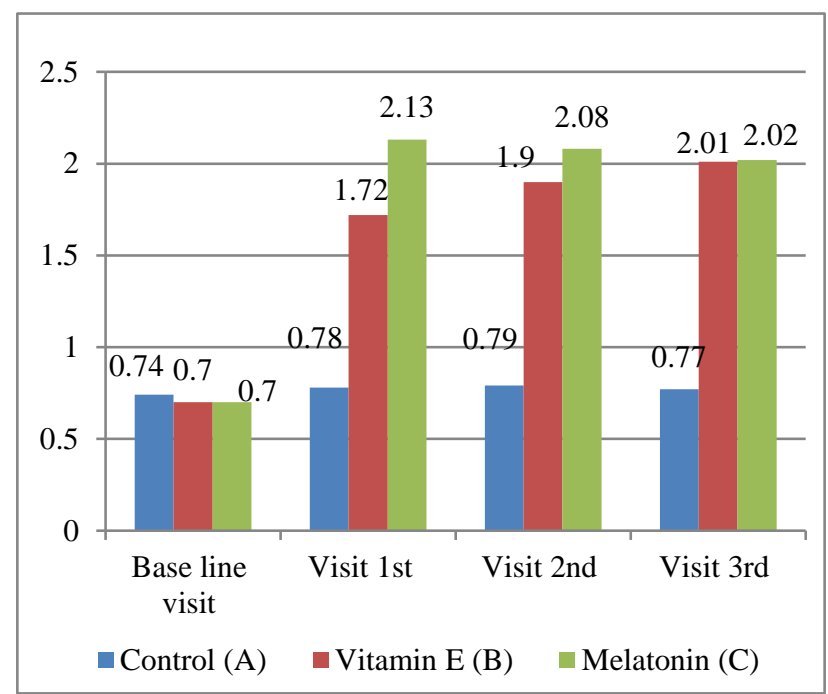

Figure 3: Comparison of mean values of glutathione peroxidase at four visits amongst all the groups.

\section{DISCUSSION}

The aim of this study was to evaluate the effects of administration of oral melatonin on the oxidative stress in periodontitis patients in rural population.

In subgroup analysis of lipid peroxidation and antioxidants level, it was seen that the study groups treated with vitamin $\mathrm{E}$ and melatonin showed a significant decrease in serum malondialdehyde (MDA) levels from baseline to posttreatment levels. High pre-treatment levels of MDA $(\mathrm{nmol} / \mathrm{ml})$ in all the groups $(4.19 \pm 0.52,4.71 \pm 1.14$ and $5.49 \pm 1.00)$ indicate the presence of oxidative stress in these patients and serves as a biomarker of lipid peroxidation. A study to evaluate the protective effect of melatonin on oxidative stress demonstrated significant decrease in values of MDA after treatment with melatonin, reflecting increased lipid peroxidation because of oxidative stress. ${ }^{11}$ There are large numbers of studies demonstrating the protective effect of melatonin on lipid peroxidation in different clinical settings.

There was a highly significant decrease in the mean values of MDA in group B when compared with group A at $3^{\text {rd }}$ visit. Mean value of MDA showed highly significant decrease in group $\mathrm{C}$ when compared with group $\mathrm{A}$ at all the visits $(\mathrm{p}<0.01)$. Group $\mathrm{C}$ showed highly significant decrease in MDA level when compared with group B at $1^{\text {st }}$ and $2^{\text {nd }}$ visits $(\mathrm{p}<0.01)$. These results show that melatonin treated group was better in reducing MDA levels as compared to both the other groups. Similarly, the study conducted by Ghosh $G$ et al states that melatonin is generally more effective than vitamin $\mathrm{E}$ for neutralizing the free radicals normally responsible for more than half of the free radical damage of the body (causing lipid peroxidation). ${ }^{12}$

Superoxide dismutase (SOD) is the antioxidant enzyme which helps to remove the superoxide radical from tissues by spontaneous dismutation to hydrogen peroxide. In oxidative stress the levels of SOD are insufficient to remove large amount of free radicals from the tissue. Our study showed that pre treatment levels of SOD were low in all the groups $(2590.33 \pm 263.96,2137.58 \pm 537.28$, $2199.50 \pm 460.03$ ) which was significantly increased in group B and C after supplementation with vitamin $\mathrm{E}$ and melatonin. It showed that there was a highly significant increase in mean values of SOD in group B and C when compared with group A $(\mathrm{p}<0.01)$ at most of the visits. But the increase in levels of SOD was highly significant in group $\mathrm{C}$ as compared to group B at $2^{\text {nd }}$ visit. Thus, our study proved that vitamin E treated group was better than control group and melatonin treated group was better than both the other groups. Similar improvement in all the antioxidant enzymes like SOD, GPx and catalase was shown by the study 'Effect of melatonin on antioxidant enzymes in human diabetic skin fibroblasts' conducted by Ewa Kilanczyk and Maria Bryszewska. ${ }^{13}$

Glutathione peroxidase (GPx) is the antioxidant enzyme present in the extracellular environment responsible for conversion of reduced glutathione (GSH) to its oxidized form (GSSG), thus reducing the free radical load in the body. Our study showed that there was a significant increase in the activity of GPx after treatment with vitamin $\mathrm{E}$ and melatonin as compared with the control group. There was a highly significant increase in the mean values of GPx in group B and C when compared with group A at all the visits, and group $\mathrm{C}$ at $1^{\text {st }}$ and $2^{\text {nd }}$ visits $(\mathrm{p}<0.01)$ when compared with group B. The study 'Melatonin increases activities of glutathione peroxidase and superoxide dismutase in fetal rat brain conducted by Okatani Y et al also states that GSH-Px activity in fetal brain homogenates increased significantly $(\mathrm{p}<0.01)$ after melatonin administration. ${ }^{14}$

Ghosh $\mathrm{G}$ et al, studied that melatonin has no morphophysiological barriers and is readily available in cytosol; whereas vitamin $\mathrm{E}$ is primarily confined to the lipid membrane. ${ }^{12}$ Thus, it is likely that melatonin is more effective antioxidant in the cytosol than vitamin $\mathrm{E}$. Melatonin is generally more effective than vitamin E, glutathione or such other antioxidants for neutralizing $\cdot \mathrm{OH}$, the free radical responsible for more than half of the free radical damage in the body. In addition to the $\mathrm{OH}$ and peroxyl radical, melatonin neutralizes superoxide, singlet oxygen, hydrogen peroxide and hypochlorous acid. Melatonin increase gene expression and activity of antioxidant enzymes GPx, SOD and catalase. Moreover, not only melatonin, but several endogenously generated metabolites of melatonin, functions as free radical scavenger. These could be some of the reasons of superiority of melatonin over vitamin E. 
The findings in our study indicate the important role played by melatonin in combating the oxidative stress in periodontitis at well tolerated doses. In view of this antioxidant action, a combination of antioxidant vitamins may also be considered in the treatment of periodontitis.

Nevertheless, the treatment duration in our study was one month and since periodontitis can recur, it would be worthwhile to conduct a study with prolonged treatment with melatonin, involving a larger sample size.

\section{CONCLUSION}

It was demonstrated that there was considerable oxidative stress in periodontitis patients, as established by high serum MDA levels, which was reduced significantly by melatonin reflecting its antioxidant potential. Pretreatment levels of Sod and GPx also were low, which were improved with the treatment of melatonin far better than with vitamin E. With the above background, it can be said that melatonin acted as an antioxidant in the patients of periodontitis which has positive effect on biochemical parameters of periodontitis, conferring a new facet to the management of periodontitis and an attempt to impede the disease progression.

Thus, this study amply justifies the role of melatonin in the control of periodontal disease and the conclusions drawn could be useful in generating further studies.

Funding: No funding sources

Conflict of interest: None declared

Ethical approval: The study was approved by the Institutional Ethics Committee of Pravara Institute of Medical Sciences, Ahmednagar, Maharashtra, India

\section{REFERENCES}

1. Chapple ILC. Role of free radicals \& antioxidants in the pathogenesis of the inflammatory periodontal diseases. J Clin Pathol. 1996;49:247-55.

2. Battino $\mathbf{M}$, Bullon $\mathrm{P}$, Wilson $\mathbf{M}$ and Newman $\mathbf{H}$. Oxidative injury and inflammatory periodontal diseases. The challenge of antioxidants to free radicals and reactive oxygen species. Crit Rev Oral Biol Med. 1999;10(4):458-76.

3. Baltacioglu E, Yuva P, Aydin G, Alver A, Kahraman C, Karabulut E, et al. Lipid peroxidation levels and total oxidant/antioxidant status in serum and saliva from patients with chronic and aggressive periodontitis. Oxidative stress index: a new biomarker for periodontal disease? J. Periodontol. 2014;85:143241.

4. Malhotra S, Sawhney G and Pandhi P. The therapeutic potential of Melatonin: A review of the Science. Med Gen Med. 2004;6(2):46.

5. Russel RJ, Dun-Xian, Juan C, Mayo. Melatonin as an antioxidant: biochemical mechanisms and pathophysological implications in humans. Acta Biochemica Polonica. 2003;50(4):1129-46.

6. Russel RJ, Corneiro RC and Oh CS. Melatonin in relation to cellular antioxidative defence mechanisms. Horm Metab Res. 1997;29(8):263-72.

7. Kantarci A, Thomas E, Van Dyke. Resolution of inflammation in Periodontitis. J Periodontal. 2005;76(11 suppl):2168-74.

8. Yagi K. Simple flurometric assay for lipid peroxidation in blood plasma. Biochem Med. 1976;15:212-5.

9. Marklund, Marklund G. Involvement of the Superoxide anion radical in the auto oxidation of pyrogallol and a convenient assay for superoxide dismutase. Eur J Biochem. 1974;47:469-74.

10. Rotruck JT, Pope AL, Ganther HE, Swanson AB, Hafeman DG, Hoekstra WG. Selenium: Biochemical role as a component of glutathione peroxidase purification and assay. Science. 1973;179:588-90.

11. Carrillo-Vico A, Calvo JR, Abreu P, Lardone PJ, García-Mauriño S, Russel RJ, et al. Evidence of melatonin synthesis by human lymphocytes and its physiological significance: Possible role as intracrine, autocrine, and/or paracrine substance. FASEB J Express. 2004;8(10):1096.

12. Ghosh G, De K, Maity S, Bandyopadhyay D, Bhattacharya S, Reiter RJ, et al. Malatonin protects against oxidative damage and restores expression of GLUT4 gene in the hyperthyroid rat heart. J Pineal Res. 2007;42(1):5-30.

13. Kilanczyk E, Bryszewska M. Effect of melatonin on antioxidant enzymes in human diabetic skin fibroblasts. Cellular Mole Biol. 2003;8:333-6.

14. Okatani Y, Wakatsuki A and Kaneda C. Melatonin increases activities of glutathione peroxidase and superoxide dismutase in fetal rat brain. J Pineal Res. 2002;28(2):89-96.

Cite this article as: Marawar A, Marawar P, Nandal DH, Kunkulol R, Narwane S. Evaluation of antioxidant potential of melatonin in periodontitis: a prospective clinic-biochemical study. Int J Basic Clin Pharmacol 2019;8:1331-5. 